\title{
A PENCIL OF ENRIQUES SURFACES WITH NON-ALGEBRAIC INTEGRAL HODGE CLASSES
}

\author{
JOHN CHRISTIAN OTTEM AND FUMIAKI SUZUKI
}

\begin{abstract}
We prove that there exists a pencil of Enriques surfaces defined over $\mathbb{Q}$ with non-algebraic integral Hodge classes of non-torsion type. This gives the first example of a threefold with the trivial Chow group of zero-cycles on which the integral Hodge conjecture fails. As an application, we construct a fourfold which gives the negative answer to a classical question of Murre on the universality of the Abel-Jacobi maps in codimension three.
\end{abstract}

\section{INTRODUCTION}

For a smooth complex projective variety $X$, we denote by $C H^{p}(X)$ the Chow group of codimension $p$ cycles and by $H^{2 p}(X, \mathbb{Z})$ the Betti cohomology group of degree $2 p$. The image $H_{\text {alg }}^{2 p}(X, \mathbb{Z}) \subseteq H^{2 p}(X, \mathbb{Z})$ of the cycle class map cl ${ }^{p}: C H^{p}(X) \rightarrow$ $H^{2 p}(X, \mathbb{Z})$ is contained in the group $H_{d g^{2 p}}(X, \mathbb{Z}) \subseteq H^{2 p}(X, \mathbb{Z})$ of integral Hodge classes. The integral Hodge conjecture is the statement that these two subgroups of $H^{2 p}(X, \mathbb{Z})$ coincide. While this statement holds for $p=0,1$ and $\operatorname{dim} X$, it is known that it can fail in general. The first counterexample was given by Atiyah-Hirzebruch [1], who constructed a projective manifold admitting a non-algebraic degree four torsion class. Later, a different type of counterexample was constructed by Kollár [2, p. 134, Lemma], who proved that for certain high degree hypersurfaces $X \subset \mathbb{P}^{4}$, the generator of $H^{4}(X, \mathbb{Z})=\mathbb{Z}$ is not algebraic. This means that the natural inclusion

$$
H_{\mathrm{alg}}^{4}(X, \mathbb{Z}) / \text { tors } \subset H d g^{4}(X, \mathbb{Z}) / \text { tors }
$$

can be strict. Since then, many other examples of non-algebraic integral Hodge classes have been found, both of torsion type [25, 5] and of non-torsion type [9, 28, 10].

In this paper, we study Enriques surface fibrations over curves and show that they can admit non-algebraic integral Hodge classes of non-torsion type.

Theorem 1.1 (=Theorem 3.1). There exists a pencil of Enriques surfaces defined over $\mathbb{Q}$ such that the cohomology groups $H^{i}(X, \mathbb{Z})$ are torsion-free for all $i$ and the inclusion

$$
H_{\mathrm{alg}}^{4}(X, \mathbb{Z}) \subsetneq H d g^{4}(X, \mathbb{Z})
$$

is strict.

One can compare Theorem 1.1 with the result of Benoist-Ottem [5], which showed that the integral Hodge conjecture can fail on products $S \times C$ for an Enriques surface $S$ and curve $C$ of genus at least one. In those examples, the non-algebraic classes in question are 2-torsion, but the integral Hodge classes are algebraic modulo torsion classes by the Künneth formula.

Theorem 1.1 also relates to certain questions concerning rational points of algebraic varieties. In a letter to Grothendieck, Serre asked whether a projective

Date: December 27, 2019.

2010 Mathematics Subject Classification. 14C25, 14C30, 14J28.

Key words and phrases. Chow groups, Hodge classes, Abel-Jacobi maps, Enriques surfaces. 
variety over the function field of a curve always has a rational point if it is $\mathcal{O}$-acyclic, that is, $H^{i}\left(X, \mathcal{O}_{X}\right)=0$ for all $i>0$. This question was answered negatively by Grabber-Harris-Mazur-Starr [12], who constructed an Enriques surface without rational points over the function field of a complex curve. Later, more explicit constructions of such Enriques surfaces were given by Lafon [17] and Starr [26].

According to [26], Esnault expected that the Enriques surfaces of [12] and [17] would satisfy a stronger property that every closed point has even degree over the base field. If that were the case, it would give a pencil of Enriques surfaces with non-algebraic integral Hodge classes of non-torsion type (this follows from [9, Theorem 7.6]). In fact, this observation was the starting point of the present paper.

Another feature of our example is that it has a trivial Chow group of zero-cycles. Indeed, Bloch-Kas-Lieberman [6] proved that $C H_{0}(S)=\mathbb{Z}$ for any Enriques surface $S$, and from this one deduces that the same holds for any pencil of Enriques surfaces (see Lemma 2.4). To our knowledge, this is the first example of a threefold with the trivial Chow group of zero-cycles on which the integral Hodge conjecture fails (see [9, Subsection 5.7] for a threefold constructed by Colliot-Thélène and Voisin which conjecturally satisfies this condition). We emphasize that it is not a priori obvious that such a threefold should exist. For instance, typical examples with the trivial Chow groups of zero-cycles are given by rationally connected varieties while the integral Hodge conjecture holds on rationally connected threefolds by a result of Voisin [30].

As an application, we settle a classical question on the universality of the AbelJacobi maps. We denote by $A^{p}(X) \subset C H^{p}(X)$ the subgroup of cycle classes algebraically equivalent to zero. The Abel-Jacobi map

$$
\psi^{p}: A^{p}(X) \rightarrow J_{a}^{p}(X),
$$

where $J_{a}^{p}(X)$ is the Lieberman intermediate Jacobian [18], is regular: it defines an invariant on $A^{p}(X)$ with values in an abelian variety such that for any algebraic family of codimension $p$ cycles on $X$, the function mapping each point of the base of the family to the value of the invariant of the corresponding codimension $p$ cycle is algebraic (see Section 4 for a more precise definition of regular homomorphisms). A classical question of Murre [22, Section 7][14, p. 132] asks whether the Abel-Jacobi map is universal among all regular homomorphisms (see [31] for another universality question from a different perspective). This is known to be true for $p=1,2$ and $\operatorname{dim} X$ [21]. Combined with [27, Theorem 1.3], Theorem 1.1 implies that the question has a negative answer in the first open case $p=3$.

Corollary 1.2 (=Corollary 4.2). Let $X$ be the pencil of Enriques surfaces of Theorem 1.1. Then there exists an elliptic curve E such that the Abel-Jacobi map

$$
\psi^{3}: A^{3}(X \times E) \rightarrow J_{a}^{3}(X \times E)
$$

is not universal: it factors through a universal regular homomorphism and the projection is an isogeny with non-zero kernel.

This paper is organized as follows. In Section 2, we study the geometry of the pencils of Enriques surfaces appearing in Theorem 1.1. These are defined as the rank one degeneracy loci of maps of vector bundles on $\mathbb{P}^{1} \times \mathbb{P}^{2} \times \mathbb{P}^{2}$. In particular, we compute their integral cohomology groups and Chow groups of zero-cycles. In Section 3 , we prove the main theorem, using a specialization argument. In Section 4, we apply the main theorem to Murre's question on the universality of the Abel-Jacobi maps.

We work over the complex numbers throughout. 
Acknowledgements. The authors would like to thank Olivier Benoist, Jørgen Vold Rennemo, Jason Starr, and Claire Voisin for interesting discussions. The second author wishes to thank his advisor Lawrence Ein for constant support and warm encouragement. JCO was supported by the Research Council of Norway project no. 250104. FS was supported by the NSF Grant No. DMS-1801870. This project started while the authors were in residence at the Mathematical Sciences Research Institute in Berkeley, California, during the Spring 2019 semester.

\section{Geometry of pencils of Enriques surfaces}

In this paper, a pencil of Enriques surfaces will mean a smooth complex threefold $\mathcal{X}$ with a fibration $\mathcal{X} \rightarrow \mathbb{P}^{1}$ over $\mathbb{P}^{1}$ whose general fibers are Enriques surfaces. In the course of the proof of Theorem 1.1, we will give a few explicit constructions of such threefolds. We start with the construction of the Enriques surfaces themselves.

We will fix the following notation ${ }^{1}$ :

- $\mathbb{P}_{A}=\mathbb{P}_{\mathbb{P}^{2} \times \mathbb{P}^{2}}(\mathcal{O}(2,0) \oplus \mathcal{O}(0,2)), E_{1}=\mathbb{P}_{\mathbb{P}^{2} \times \mathbb{P}^{2}}(\mathcal{O}(2,0)), E_{2}=\mathbb{P}_{\mathbb{P}^{2} \times \mathbb{P}^{2}}(\mathcal{O}(0,2))$

- $\mathbb{P}_{B}=\mathbb{P}_{\mathbb{P}^{2} \times \mathbb{P}^{2}}(\mathcal{O}(1,0) \oplus \mathcal{O}(0,1)), F_{1}=\mathbb{P}_{\mathbb{P}^{2} \times \mathbb{P}^{2}}(\mathcal{O}(1,0)), F_{2}=\mathbb{P}_{\mathbb{P}^{2} \times \mathbb{P}^{2}}(\mathcal{O}(0,1))$

- $\mathbb{P}_{C}=\mathbb{P}\left(H^{0}\left(\mathbb{P}_{B}, \mathcal{O}(1)\right)\right), P_{1}=\mathbb{P}\left(H^{0}\left(\mathbb{P}^{2} \times \mathbb{P}^{2}, \mathcal{O}(1,0)\right)\right), P_{2}=\mathbb{P}\left(H^{0}\left(\mathbb{P}^{2} \times \mathbb{P}^{2}, \mathcal{O}(0,1)\right)\right)$.

These spaces are related as follows. We can regard $P_{1}$ and $P_{2}$ as disjoint planes in the five-dimensional projective space $\mathbb{P}_{C}$ via the idetification

$$
H^{0}\left(\mathbb{P}_{B}, \mathcal{O}(1)\right)=H^{0}\left(\mathbb{P}^{2} \times \mathbb{P}^{2}, \mathcal{O}(1,0)\right) \oplus H^{0}\left(\mathbb{P}^{2} \times \mathbb{P}^{2}, \mathcal{O}(0,1)\right) .
$$

Then the projective bundle $\mathbb{P}_{B}$ is identified with the blow-up of $\mathbb{P}_{C}$ along the union of $P_{1}$ and $P_{2}$ with the exceptional divisors $F_{1}$ and $F_{2}$. Moreover, there is a natural involution $\iota$ on $\mathbb{P}_{C}$ induced by the involution on $H^{0}\left(\mathbb{P}_{B}, \mathcal{O}(1)\right)$ with the $( \pm 1)$-eigenspaces $H^{0}\left(\mathbb{P}^{2} \times \mathbb{P}^{2}, \mathcal{O}(1,0)\right)$ and $H^{0}\left(\mathbb{P}^{2} \times \mathbb{P}^{2}, \mathcal{O}(0,1)\right)$, respectively. The involution $\iota$ lifts to an involution on $\mathbb{P}_{B}$, and we have $\mathbb{P}_{A}=\mathbb{P}_{B} / \iota$. Thus there is a double cover $\mathbb{P}_{B} \rightarrow \mathbb{P}_{A}$ over $\mathbb{P}^{2} \times \mathbb{P}^{2}$, which is ramified along $F_{i}$, and the divisors $F_{i}$ are mapped isomorphically onto $E_{i}$ for $i=1,2$.

The projective models of the Enriques surfaces are defined as follows. On $\mathbb{P}^{2} \times \mathbb{P}^{2}$, we consider a map of vector bundles

$$
u: \mathcal{O}^{\oplus 3} \rightarrow \mathcal{O}(2,0) \oplus \mathcal{O}(0,2) .
$$

Let $X$ be the rank one degeneracy locus of $u$.

Lemma 2.1. If $u$ is general, then $X$ is an Enriques surface.

Proof. Since the vector bundle $\mathcal{O}(2,0) \oplus \mathcal{O}(0,2)$ is globally generated, $X$ is smooth of dimension two by the Bertini theorem for degeneracy loci.

To show that $X$ is an Enriques surface, we will describe its K3 cover $Y$. The map $u$ defines a global section $s$ of $\mathcal{O}(1)^{\oplus 3}$ on the projective bundle $\mathbb{P}_{A}$. When $u$ is generic, the zero set $Z(s) \subset \mathbb{P}_{A}$ maps isomorphically onto $X$ via the bundle projection $\mathbb{P}_{A} \rightarrow \mathbb{P}^{2} \times \mathbb{P}^{2}$.

On the other hand, the map $u$ also defines a global section of $\mathcal{O}(2)^{\oplus 3}$ on $\mathbb{P}_{B}$ invariant under the action of $\iota$. Indeed, as $\left(q_{*} \mathcal{O}_{\mathbb{P}_{B}}(2)\right)^{\iota}=\left(q_{*} q^{*} \mathcal{O}_{\mathbb{P}_{A}}(1)\right)^{\iota}=\mathcal{O}_{\mathbb{P}_{A}}(1)$, where $q: \mathbb{P}_{B} \rightarrow \mathbb{P}_{A}=\mathbb{P}_{B} / \iota$ is a natural projection, we have a natural identification

$$
H^{0}\left(\mathbb{P}_{B}, \mathcal{O}(2)\right)^{\iota}=H^{0}\left(\mathbb{P}_{A}, \mathcal{O}(1)\right)=H^{0}\left(\mathbb{P}^{2} \times \mathbb{P}^{2}, \mathcal{O}(2,0) \oplus \mathcal{O}(0,2)\right) .
$$

Let $Y \subset \mathbb{P}_{B}$ denote the zero set of this section. When $u$ is general, we have $X \cap E_{i}=Y \cap F_{i}=\emptyset$, so $Y$ maps isomorphically to a smooth intersection of three quadrics in $\mathbb{P}_{C}$ via the blow-down map $\mathbb{P}_{B} \rightarrow \mathbb{P}_{C}$. In particular, $Y$ is a K3 surface.

\footnotetext{
${ }^{1}$ We use Grothendieck's notation for projective bundles: for a vector bundle $\mathcal{E}, \mathbb{P}(\mathcal{E})$ paramterizes one-dimensional quotients of $\mathcal{E}$.
} 
Again since $Y \cap F_{i}=\emptyset$, the composition $\mathbb{P}_{B} \rightarrow \mathbb{P}_{A} \rightarrow \mathbb{P}^{2} \times \mathbb{P}^{2}$ restricts to an étale double cover $Y \rightarrow X$. Hence $X$ is an Enriques surface.

Remark 2.2. The proof of Lemma 2.1 shows that the construction of Enriques surfaces introduced above coincides with a classical one from [4, Example VIII.18].

We will now use a variant of the above construction to construct pencils of Enriques surfaces. On $\mathbb{P}^{1} \times \mathbb{P}^{2} \times \mathbb{P}^{2}$, we consider a map of vector bundles

$$
v: \mathcal{O}^{\oplus 3} \rightarrow \mathcal{O}(1,2,0) \oplus \mathcal{O}(1,0,2) .
$$

Let $\mathcal{X}$ be the rank one degeneracy locus of $v$.

Lemma 2.3. If $v$ is general, then $\mathcal{X}$ is a pencil of Enriques surfaces by the first projection $\mathcal{X} \rightarrow \mathbb{P}^{1}$. Moreover, we have $H^{i}\left(\mathcal{X}, \mathcal{O}_{\mathcal{X}}\right)=0$ for all $i>0$.

Proof. Since the vector bundle $\mathcal{O}(1,2,0) \oplus \mathcal{O}(1,0,2)$ is globally generated, $\mathcal{X}$ is smooth and $\operatorname{dim} \mathcal{X}=3$ by the Bertini theorem for degeneracy loci. Moreover, $\mathcal{X}$ is connected since it is defined by three equations of tridegree $(2,2,2)$. The resolution of the ideal sheaf $\mathcal{I}_{\mathcal{X}}$ of $\mathcal{X}$ in $\mathbb{P}^{1} \times \mathbb{P}^{2} \times \mathbb{P}^{2}$ has the form

$$
0 \rightarrow \mathcal{O}(-3,-4,-2) \oplus \mathcal{O}(-3,-2,-4) \rightarrow \mathcal{O}(-2,-2,-2)^{\oplus 3} \rightarrow \mathcal{I}_{\mathcal{X}} \rightarrow 0 .
$$

From this it follows that $H^{i}\left(\mathcal{X}, \mathcal{O}_{\mathcal{X}}\right)=0$ for all $i>0$.

We assume that $v$ is general in what follows.

Lemma 2.4. The degree homomorphism $\operatorname{deg}: C H_{0}(\mathcal{X}) \rightarrow \mathbb{Z}$ is an isomorphism.

Proof. Let $C \subset \mathcal{X}$ be a smooth curve which is a complete intersection of very ample divisors. Then $C H_{0}(\mathcal{X})$ is supported on $C$. This follows from the fact that any class in $\mathrm{CH}_{0}(\mathcal{X})$ is represented by a zero-cycle supported on a union of smooth fibers of the first projection $\mathcal{X} \rightarrow \mathbb{P}^{1}$ by the moving lemma, and that the Chow group of zero-cycles on any given Enriques surface is trivial due to Bloch-Kas-Lieberman [6].

We consider a natural homomorphism $\phi: \operatorname{Ker}(\operatorname{deg}) \rightarrow \operatorname{Alb}(\mathcal{X})$ induced by the Albanese map. Since $C H_{0}(\mathcal{X})$ is supported on a curve, the decomposition of the diagonal [8] implies that $\operatorname{Ker}(\phi)$ is torsion. Moreover $\operatorname{Ker}(\phi)$ is torsion-free by the Roitman theorem [23]. Hence we have $\operatorname{Ker}(\phi)=0$ and $\phi$ is an isomorphism. In our situation, $\operatorname{Alb}(\mathcal{X})=0$ since $H^{1}\left(\mathcal{X}, \mathcal{O}_{\mathcal{X}}\right)=0$ by Lemma 2.3. Therefore $\operatorname{Ker}(\operatorname{deg})=0$. The proof is complete.

To study the geometric properties of the threefold $\mathcal{X}$ in more detail, it will be convenient to involve its double cover. Recalling the construction above, we get a diagram

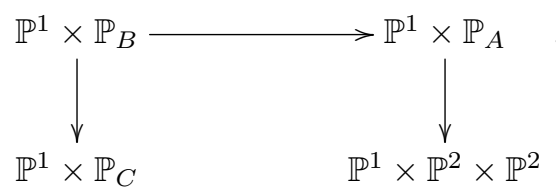

where $\mathbb{P}^{1} \times \mathbb{P}_{B} \rightarrow \mathbb{P}^{1} \times \mathbb{P}_{A}$ is the quotient by the involution $\iota$ (which acts as before on $\mathbb{P}_{B}$ and as the identity on the first factor) and $\mathbb{P}^{1} \times \mathbb{P}_{B} \rightarrow \mathbb{P}^{1} \times \mathbb{P}_{C}$ is the blow-up of $\mathbb{P}^{1} \times \mathbb{P}_{C}$ along the union of $\mathbb{P}^{1} \times P_{1}$ and $\mathbb{P}^{1} \times P_{2}$. Restricting to $\mathcal{X}$, we get the following diagram

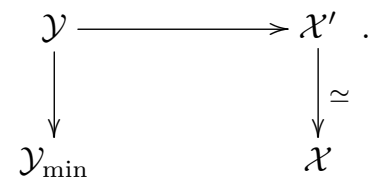


The varieties appearing in this diagram can be described as follows. The map $v$ induces a global section of $\mathcal{O}(1,1)^{\oplus 3}$ on $\mathbb{P}^{1} \times \mathbb{P}_{A}$ as well as global sections of $\mathcal{O}(1,2)^{\oplus 3}$ on $\mathbb{P}^{1} \times \mathbb{P}_{B}$ and $\mathbb{P}^{1} \times \mathbb{P}_{C}$ which are invariant under the action of $\iota$; the varieties $\mathcal{X}^{\prime}, \mathcal{Y}, \mathcal{Y}_{\min }$ are the zero sets of these sections. By generality, $\mathcal{X}^{\prime}, \mathcal{Y}, \mathcal{Y}_{\min }$ are smooth threefolds; $\mathcal{X}^{\prime}$ is mapped isomorphically onto $\mathcal{X}$, so we can identify $\mathcal{X}^{\prime}$ with $\mathcal{X} ; \mathcal{Y}$ is a double cover of $\mathcal{X}^{\prime}=\mathcal{X}$; and $\mathcal{Y}_{\text {min }}$ is a minimal model of $\mathcal{Y}$. Note that $\mathcal{Y}$ and $\mathcal{Y}_{\text {min }}$ are K3 surface fibrations via the first projection.

An easy computation shows that each of the intersections $\mathcal{Y}_{\min } \cap\left(\mathbb{P}^{1} \times P_{i}\right)$ consists of twelve points $y_{i, 1}, \cdots, y_{i, 12}$. Then the map $\mathcal{Y} \rightarrow \mathcal{Y}_{\text {min }}$ is the blow-up of $\mathcal{Y}_{\text {min }}$ along $y_{i, j}$ whose exceptional divisors $F_{i, j}$ are the components of $\mathcal{Y} \cap\left(\mathbb{P}^{1} \times F_{i}\right)$. Moreover the double cover $\mathcal{Y} \rightarrow \mathcal{X}$ is ramified along $F_{i, j}$ which are mapped isomorphically onto $E_{i, j}$, the components of $\mathcal{X} \cap\left(\mathbb{P}^{1} \times E_{i}\right)$.

Lemma 2.5. The threefold $\mathcal{X}$ has Kodaira dimension one.

Proof. Let $X$ be the class of a fiber of the first projection $\mathcal{X} \rightarrow \mathbb{P}^{1}$. It is straightforward to compute that

$$
2 K_{\mathcal{X}}=2 X+\sum_{i=1}^{2} \sum_{j=1}^{12} E_{i, j} .
$$

As the normal bundles $N_{E_{i, j} / \mathcal{X}}=\mathcal{O}_{\mathbb{P}^{2}}(-2)$ are negative, we obtain that $\kappa(\mathcal{X})=$ 1 .

Lemma 2.6. The Hodge numbers of $\mathcal{X}$ are given by $h^{0,0}(\mathcal{X})=h^{3,3}(\mathcal{X})=1$, $h^{1,1}(\mathcal{X})=h^{2,2}(\mathcal{X})=26, h^{1,2}(\mathcal{X})=h^{2,1}(\mathcal{X})=45$, and $h^{p, q}(\mathcal{X})=0$ otherwise.

Proof. We first compute the Picard number $\rho(\mathcal{X})$. Using the Lefschetz hyperplane section theorem, $\mathcal{Y}_{\text {min }}$ has Picard number two, so $\rho(\mathcal{Y})=\rho\left(\mathcal{Y}_{\min }\right)+24=26$. Moreover, the action of $\iota$ on the Picard group of $\mathcal{Y}$ is trivial, so also $\rho(\mathcal{X})=26$.

We next compute the Betti numbers $b_{i}(\mathcal{X})$. It is straightforward to compute the topological Euler characteristic $\chi_{\text {top }}(\mathcal{X})=c_{3}\left(T_{\mathcal{X}}\right)=-36$. Obviously $b_{0}(\mathcal{X})=$ $b_{6}(\mathcal{X})=1$. Moreover, $b_{1}(\mathcal{X})=b_{5}(\mathcal{X})=0$ and $b_{2}(\mathcal{X})=b_{4}(\mathcal{X})=\rho(\mathcal{X})=26$ using Lemma 2.3. Therefore $b_{3}(\mathcal{X})=90$.

Now the computation of the Hodge numbers are immediate using Lemma 2.3 again.

We next study the topology of $\mathcal{X}$. We fix the following notation:

- $\mathcal{X}_{\min }=\mathcal{Y}_{\min } / \iota$

- $\mathcal{Y}^{\circ}=\mathcal{Y}_{\min }-\left\{y_{i, j}\right\}_{i, j}$;

$-\mathcal{X}^{\circ}=\mathcal{Y}^{\circ} / \iota$

- $V_{i, j} \subset \mathcal{Y}$, a small ball around $y_{i, j}$;

- $U_{i, j}=V_{i, j} / \iota$.

We have $\mathcal{Y}_{\text {min }}=\mathcal{Y}^{\circ} \cup\left(\bigcup_{i, j} V_{i, j}\right)$ and $\mathcal{X}_{\min }=\mathcal{X}^{\circ} \cup\left(\bigcup_{i, j} U_{i, j}\right)$.

Lemma 2.7. The threefold $\mathcal{X}$ is simply connected, and the cohomology groups $H^{i}(\mathcal{X}, \mathbb{Z})$ are torsion-free for all $i$.

Proof. By the universal coefficient theorem, it is enough to prove that $\pi_{1}(\mathcal{X})=0$ and $H^{3}(\mathcal{X}, \mathbb{Z})$ is torsion-free.

We first prove that $\pi_{1}(\mathcal{X})=0$. We have a natural pushout diagram

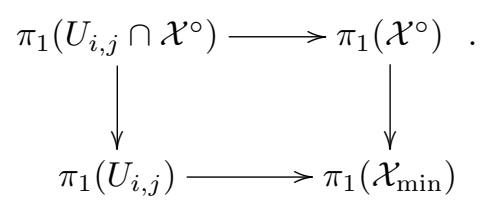


By Lefschetz, $\mathcal{Y}$ and hence $\mathcal{Y}^{\circ}$ is simply connected. So since the quotient map $\pi: \mathcal{Y}^{\circ} \rightarrow \mathcal{X}^{\circ}$ is étale, we have $\pi_{1}\left(\mathcal{X}^{\circ}\right)=\mathbb{Z} / 2$. The neighbourhood $U_{i, j} \subset \mathcal{X}$ is homotopic to the affine cone over a Veronese surface, so we have $\pi_{1}\left(U_{i, j}\right)=0$. Finally, since the map $V_{i, j} \cap \mathcal{Y}^{\circ} \rightarrow U_{i, j} \cap \mathcal{X}^{\circ}$ is homotopic to the universal covering $\operatorname{map}\left(\mathbb{C}^{3}-0\right) \rightarrow\left(\mathbb{C}^{3}-0\right) / \pm$, we have $\pi_{1}\left(U_{i, j} \cap \mathcal{X}^{\circ}\right)=\mathbb{Z} / 2$. In fact, this cover is induced by the restriction of $\pi$ to $V_{i, j} \cap \mathcal{Y}^{\circ}$, so the map $\pi_{1}\left(U_{i, j} \cap \mathcal{X}^{\circ}\right) \rightarrow \pi_{1}\left(\mathcal{X}^{\circ}\right)$ is non-zero, hence an isomorphism. From the pushout diagram above, we then get $\pi_{1}\left(\mathcal{X}_{\text {min }}\right)=0$. Resolving a finite cyclic quotient singularity does not change the fundamental group $\left(\left[16\right.\right.$, Theorem 7.8]), so we also get $\pi_{1}(\mathcal{X})=0$.

We next prove that $H^{3}(\mathcal{X}, \mathbb{Z})$ is torsion-free. The long exact sequence for cohomology groups with supports gives

$$
\bigoplus_{i, j} H_{E_{i, j}}^{3}(\mathcal{X}, \mathbb{Z}) \rightarrow H^{3}(\mathcal{X}, \mathbb{Z}) \rightarrow H^{3}\left(\mathcal{X}^{\circ}, \mathbb{Z}\right)
$$

Since $H_{E_{i, j}}^{3}(\mathcal{X}, \mathbb{Z})=H_{3}\left(E_{i, j}, \mathbb{Z}\right)=0$, the group $H^{3}(\mathcal{X}, \mathbb{Z})$ injects into $H^{3}\left(\mathcal{X}^{\circ}, \mathbb{Z}\right)$. In particular, we are reduced to showing that $H^{3}\left(\mathcal{X}^{\circ}, \mathbb{Z}\right)$ is torsion-free.

Since $\mathcal{X}^{\circ}$ is the quotient of $\mathcal{Y}^{\circ}$ by the group $\langle\iota\rangle \simeq \mathbb{Z} / 2$, we can apply the Cartan-Leray spectral sequence

$$
E_{2}^{p, q}=H^{p}\left(\mathbb{Z} / 2, H^{q}\left(\mathcal{Y}^{\circ}, \mathbb{Z}\right)\right) \Rightarrow H^{p+q}\left(\mathcal{X}^{\circ}, \mathbb{Z}\right)
$$

to compute the cohomology groups of $\mathcal{X}^{\circ}$. We need to compute $H^{q}\left(\mathcal{Y}^{\circ}, \mathbb{Z}\right)$ for $0 \leq q \leq 3$ and the action of $\iota$ on these groups. Since $\mathcal{Y}^{\circ}$ is obtained from $\mathcal{Y}_{\text {min }}$ by removing finitely many points, we have an identification $H^{q}\left(\mathcal{Y}^{\circ}, \mathbb{Z}\right)=H^{q}\left(\mathcal{Y}_{\min }, \mathbb{Z}\right)$. Clearly $H^{0}\left(\mathcal{Y}_{\text {min }}, \mathbb{Z}\right)=\mathbb{Z}$. By the Lefschetz hyperplane theorem, $H^{1}\left(\mathcal{Y}_{\min }, \mathbb{Z}\right)=0$, and the groups $H^{2}\left(\mathcal{Y}_{\min }, \mathbb{Z}\right)$ and $H^{3}\left(\mathcal{Y}_{\min }, \mathbb{Z}\right)$ are torsion-free. Moreover, the action of $\iota$ on $H^{q}\left(\mathcal{Y}_{\min }, \mathbb{Z}\right)$ is trivial for $0 \leq q \leq 2$. Since the group cohomology $H^{p}(\mathbb{Z} / 2, \mathbb{Z})=0$ for $p$ odd, it follows that $E_{2}^{p, 3-\bar{p}}=0$ for $p \neq 0$. Therefore there is an injection

$$
H^{3}\left(\mathcal{X}^{\circ}, \mathbb{Z}\right) \hookrightarrow E_{2}^{0,3}=H^{0}\left(\mathbb{Z} / 2, H^{3}\left(\mathcal{Y}^{\circ}, \mathbb{Z}\right)\right)=H^{3}\left(\mathcal{Y}^{\circ}, \mathbb{Z}\right)^{\iota},
$$

where the right hand side is torsion-free. This completes the proof.

\section{Proof of Theorem 1.1}

We are now ready to prove our main result:

Theorem 3.1. There exists a map of vector bundles on $\mathbb{P}^{1} \times \mathbb{P}^{2} \times \mathbb{P}^{2}$

$$
\mathcal{O}^{\oplus 3} \rightarrow \mathcal{O}(1,2,0) \oplus \mathcal{O}(1,0,2)
$$

defined over $\mathbb{Q}$ such that the rank one degeneracy locus $\mathcal{X}$ is a pencil of Enriques surfaces such that the cohomology groups $H^{i}(\mathcal{X}, \mathbb{Z})$ are torsion-free for all $i$ and there is a strict inclusion

$$
H_{\mathrm{alg}}^{4}(\mathcal{X}, \mathbb{Z}) \subsetneq H d g^{4}(\mathcal{X}, \mathbb{Z})
$$

Proof. We set $\mathbb{P}^{1} \times \mathbb{P}^{2} \times \mathbb{P}^{2}=\operatorname{Proj} \mathbb{C}[S, T] \times \operatorname{Proj} \mathbb{C}\left[X_{0}, X_{1}, X_{2}\right] \times \operatorname{Proj} \mathbb{C}\left[Y_{0}, Y_{1}, Y_{2}\right]$. Fix a sufficiently large prime number $p$. We consider a map of vector bundles as above given by the matrix

$$
M=\left(\begin{array}{ccc}
P_{1} & Q_{1} & R_{1} \\
S P_{2}+p P_{3} & S Q_{2}+p Q_{3} & S R_{2}+p R_{3}
\end{array}\right),
$$

where $P_{1}, Q_{1}, R_{1}$ (resp. $P_{2}, Q_{2}, R_{2} ; P_{3}, Q_{3}, R_{3}$ ) are general tri-homogeneous polynomials of tri-degree $(1,2,0)$ (resp. $(0,0,2) ;(1,0,2))$ over $\mathbb{Q}$. The degeneracy locus $\mathcal{X}$ is a pencil of Enriques surfaces defined by the $2 \times 2$-minors of $M$. The torsion-freeness of the cohomology groups follows from Lemma 2.7, so it remains to prove that the integral Hodge conjecture does not hold on $\mathcal{X}$. 
The closed subscheme defined by $P_{1}=Q_{1}=R_{1}=0$ is a disjoint union of twelve components $E_{1,1}, \ldots, E_{1,12}$ isomorphic to $\mathbb{P}^{2}$. We note that this union is defined over $\mathbb{Q}$, even though each $E_{i, j}$ may not be. First we prove that for a given algebraic one-cycle $\alpha$ on $\mathcal{X}$, we have

$$
\operatorname{deg}\left(\alpha / \mathbb{P}^{1}\right) \equiv \alpha \cdot\left(\sum_{j=1}^{12} E_{1, j}\right) \quad \bmod 2 .
$$

We use a specialization argument. We spread out $\mathcal{X}_{\overline{\mathbb{Q}}}$ over a valuation ring $R$ with the maximal ideal containing $p$. The ideal of the flat closure of $\mathcal{X}_{\overline{\mathbb{Q}}}$ in $\left(\mathbb{P}^{1} \times \mathbb{P}^{2} \times \mathbb{P}^{2}\right)_{R}$ is generated by the $2 \times 2$-minors of $M$ and

$$
F=\operatorname{det}\left(\begin{array}{ccc}
P_{1} & Q_{1} & R_{1} \\
P_{2} & Q_{2} & R_{2} \\
P_{3} & Q_{3} & R_{3}
\end{array}\right)
$$

The specialization over $\overline{\mathbb{F}}_{p}$ consists of two components: one is a pencil of Enriques surfaces $\widetilde{\mathcal{X}}_{0}$ defined by the $2 \times 2$-minors of the matrix

$$
N=\left(\begin{array}{lll}
P_{1} & Q_{1} & R_{1} \\
P_{2} & Q_{2} & R_{2}
\end{array}\right)
$$

the other is defined by $S=F=0$. It is straightforward to check that $\widetilde{\mathcal{X}}_{0}$ is smooth.

The closed subscheme defined by $P_{1}=Q_{1}=R_{1}=0$ is again a disjoint union of twelve components $E_{1,1}, \ldots, E_{1,12}$ isomorphic to $\mathbb{P}^{2}$ and disjoint from the fiber over $S=0$ by the generality of $P_{1}, Q_{1}, R_{1}$. We prove that for a given one-cycle $\alpha_{0}$ on the specialization over $\overline{\mathbb{F}}_{p}$, we have

$$
\operatorname{deg}\left(\alpha_{0} / \mathbb{P}^{1}\right) \equiv \alpha_{0} \cdot\left(\sum_{j=1}^{12} E_{1, j}\right) \quad \bmod 2 .
$$

We may assume that $\alpha_{0}$ is supported on $\widetilde{\mathcal{X}}_{0}$. Let $D_{1}$ be the Cartier divisor on $\widetilde{\mathcal{X}}_{0}$ defined by $P_{1}=0$. Since $D_{1}$ is of type $(1,2,0)$, we have

$$
\operatorname{deg}\left(\alpha_{0} / \mathbb{P}^{1}\right) \equiv \alpha_{0} \cdot D_{1} \quad \bmod 2
$$

On the other hand, we have

$$
D_{1}=D_{2}+\sum_{j=1}^{12} E_{1, j}
$$

where $D_{2}$ is the Cartier divisor on $\widetilde{\mathcal{X}}_{0}$ defined by $P_{2}=0$. Indeed, expanding the $2 \times 2$-minors of $N$, it is easily seen that the identity holds on each of the open subsets $P_{2}, Q_{2}, R_{2} \neq 0$; these open subsets form an open cover of $\widetilde{\mathcal{X}}_{0}$ by the generality of $P_{2}, Q_{2}, R_{2}$. Since $D_{2}$ is of type $(0,0,2)$, we have

$$
\alpha_{0} \cdot D_{1} \equiv \alpha_{0} \cdot\left(\sum_{j=1}^{12} E_{1, j}\right) \quad \bmod 2 .
$$

The congruence (2) follows, so does the congruence (1) by the specialization homomorphism [11, Section 20.3].

The Hodge structure of $H^{4}(\mathcal{X}, \mathbb{Z})$ is trivial since we have $H^{2}\left(\mathcal{X}, \mathcal{O}_{\mathcal{X}}\right)=0$ by Lemma 2.3. The proof of the theorem is reduced to proving that there exists a class 
$\beta \in H^{4}(\mathcal{X}, \mathbb{Z})=H_{2}(\mathcal{X}, \mathbb{Z})$ such that

$$
\operatorname{deg}\left(\beta / \mathbb{P}^{1}\right)= \pm 1, \beta \cdot\left(\sum_{j=1}^{12} E_{1, j}\right)=0 ;
$$

such $\beta$ is not algebraic according to the congruence (1). Since $E_{1,1}, \ldots, E_{1,12}$ are the images of $F_{1,1}, \ldots, F_{1,12}$ under the double cover $\mathcal{Y} \rightarrow \mathcal{X}$, it is enough to prove that there exists $\gamma \in H^{4}(\mathcal{Y}, \mathbb{Z})=H_{2}(\mathcal{Y}, \mathbb{Z})$ such that

$$
\operatorname{deg}\left(\gamma / \mathbb{P}^{1}\right)= \pm 1, \gamma \cdot\left(\sum_{j=1}^{12} F_{1, j}\right)=0 ;
$$

the class $\beta$ will be the push-forward of $\gamma$. By the Lefschetz hyperplane section theorem, the push-forward $H_{2}\left(\mathcal{Y}_{\min }, \mathbb{Z}\right) \rightarrow H_{2}\left(\mathbb{P}^{1}, \mathbb{Z}\right)$ is surjective. Let $\gamma_{\min } \in$ $H^{4}\left(\mathcal{Y}_{\text {min }}, \mathbb{Z}\right)=H_{2}\left(\mathcal{Y}_{\text {min }}, \mathbb{Z}\right)$ be an element mapped to a generator of $H_{2}\left(\mathbb{P}^{1}, \mathbb{Z}\right)$. Then the pullback $\gamma \in H^{4}(\mathcal{Y}, \mathbb{Z})$ of $\gamma_{\min }$ satisfies the desired property. The proof is complete.

Remark 3.2. The specialization used in the proof of Theorem 3.1 deserves a few more comments. The specialization consists of two components: $\widetilde{\mathcal{X}}_{0}$ defined by the $2 \times 2$-minors of $N$, and $R$ defined by $S=F=0$. The component $\widetilde{\mathcal{X}}_{0}$ is smooth, and it is a pencil of Enriques surfaces by the first projection $\widetilde{\mathcal{X}}_{0} \rightarrow \mathbb{P}^{1}$. On the other hand, $R$ has isolated singularities, and a smooth model $\bar{R}$ of $R$ is another pencil of Enriques surfaces with a small contraction $\bar{R} \rightarrow R$ contracting $\mathbb{P}^{1}$ s over the singular points of $R$. In addition, $\widetilde{\mathcal{X}}_{0}$ and $R$ intersect in a fiber over $S=0$, and the intersection is an Enriques surface $Z$ in $\mathbb{P}^{2} \times \mathbb{P}^{2}$.

Remarkably, both of the components $\widetilde{\mathcal{X}}_{0}$ and $R$ are rationally connected: the projections

$$
\widetilde{\mathcal{X}}_{0} \hookrightarrow \mathbb{P}^{1} \times \mathbb{P}^{2} \times \mathbb{P}^{2} \stackrel{p r_{2}}{\longrightarrow} \mathbb{P}^{2}, R \hookrightarrow \mathbb{P}^{1} \times \mathbb{P}^{2} \times \mathbb{P}^{2} \stackrel{p r_{3}}{\longrightarrow} \mathbb{P}^{2}
$$

are conic bundles, therefore this follows from [13, Corollary 1.3]. In particular, the integral Hodge conjecture holds on $\widetilde{\mathcal{X}}_{0}$ and $\bar{R}$ by a result of Voisin [30]. As a consequence, $H_{2}\left(\widetilde{\mathcal{X}}_{0}, \mathbb{Z}\right)$ and $H_{2}(R, \mathbb{Z})$ are generated by algebraic cycles.

It turns out, however, that this is not the case for the union $\widetilde{\mathcal{X}}_{0} \cup R$. A key point here is the subtle difference between the Mayer-Vietoris sequence for homology groups and Chow groups. For the homology groups, we have an exact sequence

$$
H_{2}\left(\widetilde{\mathcal{X}}_{0}, \mathbb{Z}\right) \oplus H_{2}(R, \mathbb{Z}) \rightarrow H_{2}\left(\widetilde{\mathcal{X}}_{0} \cup R, \mathbb{Z}\right) \rightarrow H_{1}(Z, \mathbb{Z})=\mathbb{Z} / 2 \rightarrow 0 .
$$

For the Chow groups, on the other hand, we obviously have a surjection

$$
C H_{1}\left(\widetilde{\mathcal{X}}_{0}\right) \oplus C H_{1}(R) \rightarrow C H_{1}\left(\widetilde{\mathcal{X}}_{0} \cup R\right)
$$

(see also [11, Example 1.8.1]). It follows that $H_{2}\left(\widetilde{\mathcal{X}}_{0} \cup R, \mathbb{Z}\right)$ is not generated by algebraic cycles.

A small modification of the above arguments yields a generalization of Theorem 3.1 to higher dimensions:

Theorem 3.3. For a given positive integer $n$, there exists a map of vector bundles on $\mathbb{P}^{1} \times \mathbb{P}^{2 n} \times \mathbb{P}^{2 n}$

$$
\mathcal{O}^{\oplus(2 n+1)} \rightarrow \mathcal{O}(1,2,0) \oplus \mathcal{O}(1,0,2)
$$

defined over $\mathbb{Q}$ such that the rank one degeneracy locus $\mathcal{X}$ is a smooth $(2 n+1)$-fold with a fibration over $\mathbb{P}^{1}$ whose general fibers are $2 n$-folds $X$ with $H^{i}\left(X, \mathcal{O}_{X}\right)=0$ for all $i>0$ and universal Calabi-Yau double covers $Y \rightarrow X$ such that 
(i) $H^{i}\left(\mathcal{X}, \mathcal{O}_{\mathcal{X}}\right)=0$ for all $i>0$;

(ii) $\kappa(\mathcal{X})=1$;

(iii) $\mathcal{X}$ is simply connected, and the cohomology group $H^{3}(\mathcal{X}, \mathbb{Z})$ is torsion-free;

(iv) the inclusion $H_{2, \text { alg }}(\mathcal{X}, \mathbb{Z}) \subsetneq H d g_{2}(\mathcal{X}, \mathbb{Z})$ is strict.

\section{Application to the universality of the Abel-Jacobi maps}

Let $V$ be a smooth complex projective variety. For an integer $p$, we let $A^{p}(V) \subseteq$ $C H^{p}(V)$ denote the subgroup of cycles algebraically equivalent to zero. We recall that a homomorphism $\phi: A^{p}(V) \rightarrow A$ to an abelian variety $A$ is called regular if for any smooth connected projective variety $S$ with a base point $s_{0}$ and for any codimension $p$ cycle $\Gamma$ on $S \times V$, the composition

$$
S \rightarrow A^{p}(V) \rightarrow A, s \mapsto \phi\left(\Gamma_{*}\left(s-s_{0}\right)\right)
$$

is a morphism of algebraic varieties (this definition goes back to the work of Samuel [24]). An important example of such homomorphisms is the following. We consider the Abel-Jacobi map

$$
A J^{p}: C H^{p}(V)_{\mathrm{hom}} \rightarrow J^{p}(V),
$$

where $C H^{p}(V)_{\text {hom }} \subset C H^{p}(V)$ is the subgroup of cycle classes homologous to zero, and

$$
J^{p}(V)=H^{2 p-1}(V, \mathbb{C}) /\left(H^{2 p-1}(V, \mathbb{Z}(p))+F^{p} H^{2 p-1}(V, \mathbb{C})\right)
$$

is the $p$-th Griffiths intermediate Jacobian (see [29, Section 12] for the definition and properties of the Abel-Jacobi maps). Then the image $J_{a}^{p}(V) \subset J^{p}(V)$ of the restriction of the Abel-Jacobi map $A J^{p}$ to $A^{p}(V)$ is an abelian variety, and the induced map

$$
\psi^{p}: A^{p}(V) \rightarrow J_{a}^{p}(V),
$$

which we also call Abel-Jacobi, is regular [15][18]. A classical question of Murre [22, Section 7][14, p. 132] asks whether the Abel-Jacobi map $\psi^{p}: A^{p}(V) \rightarrow J_{a}^{p}(V)$ is universal among all regular homomorphisms $\phi: A^{p}(V) \rightarrow A$, that is, whether every such $\phi$ factors through $\psi^{p}$. This is known to hold for $p=1$ by the theory of the Picard variety, for $p=\operatorname{dim} V$ by the theory of the Albanese variety, and for $p=2$ as proved by Murre [20][21] using the Merkurjev-Suslin theorem [19].

Meanwhile, it was proved by Walker [32] that the Abel-Jacobi map $\psi^{p}$ factors as

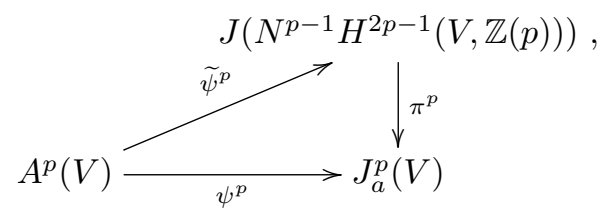

where $J\left(N^{p-1} H^{2 p-1}(V, \mathbb{Z}(p))\right)$ is the intermediate Jacobian for the mixed Hodge structure given by the coniveau filtration $N^{p-1} H^{2 p-1}(V, \mathbb{Z}(p))[7], \pi^{p}$ is a natural isogeny, and $\widetilde{\psi}^{p}$ is a surjective regular homomorphism. If the Abel-Jacobi map $\psi^{p}$ is universal, then the kernel

$\operatorname{Ker}\left(\pi^{p}\right)=\operatorname{Coker}\left(H^{2 p-1}(V, \mathbb{Z}(p))_{\text {tors }} \rightarrow\left(H^{2 p-1}(V, \mathbb{Z}(p)) / N^{p-1} H^{2 p-1}(V, \mathbb{Z}(p))\right)_{\text {tors }}\right)$

is trivial. In other words, the sublattice

$$
N^{p-1} H^{2 p-1}(V, \mathbb{Z}(p)) / \text { tors } \subset H^{2 p-1}(V, \mathbb{Z}(p)) / \text { tors }
$$

is primitive.

We recall the main theorem of the paper [27]. 
Theorem 4.1 ([27], Theorem 1.3). Let $W$ be a smooth projective variety such that $\mathrm{CH}_{0}(W)$ is supported on a surface and the inclusion

$$
H_{\text {alg }}^{4}(W, \mathbb{Z}) / \text { tors } \subsetneq H d g^{4}(W, \mathbb{Z}) / \text { tors }
$$

is strict. Then there exists an elliptic curve E such that the sublattice

$$
N^{2} H^{5}(W \times E, \mathbb{Z}(3)) / \text { tors } \subset H^{5}(W \times E, \mathbb{Z}(3)) / \text { tors }
$$

is not primitive.

Now we prove that the Abel-Jacobi map is not universal in general. This settles Murre's question.

Corollary 4.2. Let $\mathcal{X}$ be the pencil of Enriques surfaces of Theorem 3.1. Then there exists an elliptic curve E such that the Abel-Jacobi map

$$
\psi^{3}: A^{3}(\mathcal{X} \times E) \rightarrow J_{a}^{3}(\mathcal{X} \times E)
$$

is not universal: it factors as

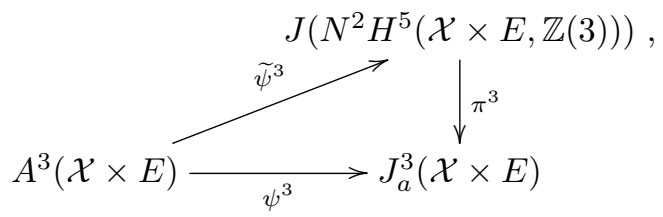

where the Walker map $\widetilde{\psi}^{3}$ is surjective regular, and the natural isogeny $\pi^{3}$ has non-zero kernel, or equivalently, the sublattice

$$
N^{2} H^{5}(\mathcal{X} \times E, \mathbb{Z}(3)) \subset H^{5}(\mathcal{X} \times E, \mathbb{Z}(3))
$$

is not primitive.

Remark 4.3. The Walker map $\widetilde{\psi}^{3}$ in the statement is universal by [27, Theorem 1.1].

Remark 4.4. In fact, we have $N^{2} H^{5}(\mathcal{X} \times E, \mathbb{Q})=H^{5}(\mathcal{X} \times E, \mathbb{Q})$ as a consequence of decomposition of the diagonal [8]. In other words, $J_{a}^{3}(\mathcal{X} \times E)=J^{3}(\mathcal{X} \times E)$ (see [22, Lemma 4.3]).

Proof of Corollary 4.2. We have $C H_{0}(\mathcal{X})=\mathbb{Z}$ by Lemma 2.4. Moreover, the cohomology group $H^{4}(\mathcal{X}, \mathbb{Z})$ is torsion-free and the inclusion $H_{\text {alg }}^{4}(\mathcal{X}, \mathbb{Z}) \subsetneq H d g^{4}(\mathcal{X}, \mathbb{Z})$ is strict by Theorem 3.1. Now the assertion follows by applying Theorem 4.1 to $W=\mathcal{X}$. The proof is complete.

Finally, we explain how to produce counterexamples to Murre's question in higher dimensions and for other values of $p$. We take $\mathcal{X}$ and $E$ as in Corollary 4.2, and let $d \geq 4$. Then, on the $d$-fold $\mathcal{X} \times E \times \mathbb{P}^{d-4}$, for all $3 \leq p \leq d-1$, the sublattice

$$
N^{p-1} H^{2 p-1}\left(\mathcal{X} \times E \times \mathbb{P}^{d-4}, \mathbb{Z}(p)\right) \subset H^{2 p-1}\left(\mathcal{X} \times E \times \mathbb{P}^{d-4}, \mathbb{Z}(p)\right)
$$

is not primitive (this follows from the formula [3, Theorem 3.1] for the Bloch-Ogus spectral sequence [7] under taking the product with a projective space). In particular, for all $3 \leq p \leq d-1$, the Abel-Jacobi map

$$
\psi^{p}: A^{p}\left(\mathcal{X} \times E \times \mathbb{P}^{d-4}\right) \rightarrow J_{a}^{p}\left(\mathcal{X} \times E \times \mathbb{P}^{d-4}\right)
$$

is not universal. 


\section{REFERENCES}

[1] Atiyah, M. F., Hirzebruch, F.: Analytic cycles on complex manifolds, Topology 1 (1962), $25-45$.

[2] Ballico, E., Catanese, F., Ciliberto, C. (eds): Classification of irregular varieties, Lecture Notes in Mathematics, 1515, Springer-Verlag, Berlin, 1992.

[3] Barbieri-Viale, L.: $\mathcal{H}$-cohomologies versus algebraic cycles, Math. Nachr. 184 (1997), 5-57.

[4] Beauville, A.: Complex algebraic surfaces, translated from the 1978 French original by R. Barlow, with assistance from N. I. Shepherd-Barron and M. Reid, second edition, London Mathematical Society Student Texts, 34, Cambridge University Press, Cambridge, 1996.

[5] Benoist, O., Ottem, J. C.: Failure of the integral Hodge conjecture for threefolds of Kodaira dimension zero, to appear in Commentarii Mathematici Helvetici (2018).

[6] Bloch, S.; Kas, A.; Lieberman, D.: Zero cycles on surfaces with $p_{g}=0$, Compositio Math. 33 (1976), no. 2, 135-145.

[7] Bloch, S., Ogus, A.: Gersten's conjecture and the homology of schemes, Ann. Sci. École Norm. Sup. (4) 7 (1974), 181-201 (1975).

[8] Bloch, S., Srinivas, V.: Remarks on correspondences and algebraic cycles, Amer. J. Math. 105 (1983), no. 5, 1235-1253.

[9] Colliot-Thélène, J. -L., Voisin, C.: Cohomologie non ramifiée et conjecture de Hodge entière, Duke Math. J. 161 (2012), no. 5, 735-801.

[10] Diaz, H. A.: On the unramified cohomology of certain quotient varieties, arXiv:1906.06598.

[11] Fulton, W.: Intersection theory, second edition, Ergebnisse der Mathematik und ihrer Grenzgebiete. 3. Folge. A Series of Modern Surveys in Mathematics, 2, Springer, Berlin, 1998.

[12] Graber, T., Harris, J., Mazur, B., Starr, J.: Rational connectivity and sections of families over curves, Ann. Sci. École Norm. Sup. (4) 38 (2005), no. 5, 671-692.

[13] Graber, T., Harris, J., Starr, J.: Families of rationally connected varieties, J. Amer. Math. Soc. 16 (2003), no. 1, 57-67.

[14] Green, M., Murre, J., Voisin, C.: Algebraic cycles and Hodge theory, Lectures given at the Second C.I.M.E. Session held in Torino, June 21-29, 1993. Edited by A. Albano and F. Bardelli. Lecture Notes in Mathematics, 1594. Springer-Verlag, Berlin, 1994. viii+275 pp.

[15] Griffiths, P. A.: Periods of integrals on algebraic manifolds. II. Local study of the period mapping, Amer. J. Math. 90 (1968), 805-865.

[16] Kollár, J.: Shafarevich maps and plurigenera of algebraic varieties, Inventiones mathematicae 113.1 (1993): 177-215.

[17] Lafon, G.: Une surface d'Enriques sans point sur $\mathbb{C}((t))$, C. R. Math. Acad. Sci. Paris 338 (2004), no. 1, 51-54.

[18] Lieberman, D.: Intermediate Jacobians, in Algebraic geometry, Oslo 1970 (Proc. Fifth Nordic Summer-School in Math.), 125-139, Wolters-Noordhoff, Groningen.

[19] Merkurjev, A. S., Suslin, A.: K-cohomology of Severi-Brauer varieties and the norm residue homomorphism, Izv. Akad. Nauk SSSR Ser. Mat. 46 (1982), no. 5, 1011-1046, 1135-1136.

[20] Murre, J. P.: Un résultat en théorie des cycles algébriques de codimension deux, C. R. Acad. Sci. Paris Sér. I Math. 296 (1983), no. 23, 981-984.

[21] Murre, J. P.: Applications of algebraic K-theory to the theory of algebraic cycles, in Algebraic geometry, Sitges (Barcelona), 1983, 216-261, Lecture Notes in Math., 1124, Springer, Berlin.

[22] Murre, J. P.: Abel-Jacobi equivalence versus incidence equivalence for algebraic cycles of codimension two, Topology 24 (1985), no. 3, 361-367.

[23] Roitman, A. A.: The torsion of the group of 0-cycles modulo rational equivalence, Ann. of Math. (2) 111 (1980), no. 3, 553-569.

[24] Samuel, P.: Relations d'équivalence en géométrie algébrique, in Proc. Internat. Congress Math. 1958, 470-487, Cambridge Univ. Press, New York.

[25] Soulé, C.; Voisin, C.: Torsion cohomology classes and algebraic cycles on complex projective manifolds, Adv. Math. 198 (2005), no. 1, 107-127.

[26] Starr, J. M.: A pencil of Enriques surfaces of index one with no section, Algebra Number Theory 3 (2009), no. 6, 637-652.

[27] Suzuki, F.: A remark on a 3-fold constructed by Colliot-Thélène and Voisin, to appear in Math. Res. Lett.

[28] Totaro, B.: On the integral Hodge and Tate conjectures over a number field, Forum Math. Sigma 1 (2013), e4, 13 pp.

[29] Voisin, C: Hodge theory and complex algebraic geometry. I, translated from the French original by Leila Schneps, Cambridge Studies in Advanced Mathematics, 76, Cambridge University Press, Cambridge, 2002. 
[30] Voisin, C.: On integral Hodge classes on uniruled or Calabi-Yau threefolds, Moduli spaces and arithmetic geometry, 43-73, Adv. Stud. Pure Math., 45, Math. Soc. Japan, Tokyo, 2006.

[31] Voisin, C.: Unirational threefolds with no universal codimension 2 cycle, Invent. Math. 201 (2015), no. 1, 207-237.

[32] Walker, M. E.: The morphic Abel-Jacobi map, Compos. Math. 143 (2007), no. 4, 909-944.

Department of Mathematics, University of Oslo, Box 1053, Blindern, 0316 Oslo, NORWAY

Email address: johnco@math.uio.no

Department of Mathematics, University of Illinois at Chicago, 851 S. Morgan Street, Chicago, IL, USA

Email address: fsuzuk2@uic.edu 